Timo Meynhardt

\title{
Gemeinwohl in Bewegung: Die Pandemie als Katalysator
}

\begin{abstract}
»One thing the coronavirus crisis has already proved is that there really is such a thing as society. ${ }^{\mathrm{I}}$ Der britische Premier Boris Johnson spricht aus, was offensichtlich ist: Es geht nur gemeinsam, das kollektive Überleben erfordert Solidarität und die Mitwirkung aller. Mit diesem Satz zu Beginn der COVID-ı-Pandemie im März 2020 bringt Johnson allerdings gleichzeitig etwas ins Rollen, was als intellektuelle Zäsur mit offenem Ausgang verstanden werden kann.

Dazu ein Rückblick: Die damalige britische Premierministerin Margaret Thatcher hatte 1987 in einem Interview den berühmt-berüchtigt gewordenen Satz »There is no such thing as society « formuliert. Aus dem Kontext des deutlich nuancierteren Interviews gerissen, wurde dieser Satz zur Signatur ihrer Amtszeit und zur Kurzformel der Akzentverschiebung hin zu mehr Selbstverantwortung des Einzelnen und weg von staatlicher Unterstützung. Je nach ideologischer Ausrichtung wurde der Grundgedanke zum Baustein politischer Programme und wirkt bis heute nach.

Ganz sicher würde sich Thatcher nicht mit jeder Auslegung ihrer Worte einverstanden erklären. Eines aber darf man ihr unterstellen: Sie war fest davon überzeugt, dass dem Gemeinwohl (sie nannte es »flourishing
\end{abstract}


society «) am besten damit gedient sei, wenn jeder für sich selbst und sein unmittelbares Umfeld Verantwortung übernimmt. Die Pandemie macht nun deutlich, wie voraussetzungsreich eine solcherart liberale Perspektive ist.

Schon jetzt ist absehbar, dass die Auswirkungen der Pandemie sich auf die ganze Gesellschaft erstrecken und nicht nur einzelne Bereiche erfassen. Es werden neue Gemeinwohlkonstellationen entstehen, die im besten Fall die Gesellschaft als Ganze resilienter, widerstandsfähiger und lebensfähiger machen. Im Folgenden möchte ich aufzeigen, welche Anstöße für das Gemeinwohldenken durch die Pandemie sich bereits abzeichnen und wie diese produktiv genutzt werden können.

\section{Was die Pandemie zeigt}

Das erste Jahr der COVID-I9-Krise führt uns (wieder einmal) vor Augen, wie abhängig wir alle von einem funktionierenden Gemeinwesen sind. Die Idee des autonomen Individuums, das seine Ziele verfolgt, für sich und andere Verantwortung übernimmt, erweist sich als wenig tragfähig, wenn die dafür notwendigen Voraussetzungen gegenseitiger Abhängigkeiten nicht beachtet werden. Genau dies ist der berechtigte Vorwurf an einen Individualismus, der in erster Linie auf Freiheitsrechten beharrt, ohne die dafür erforderliche soziale Verbundenheit (»Sozialität«) zu erkennen und zu würdigen.

Die Qualität der Beziehungen (»Verbundenheiten«) in Familie, am Arbeitsplatz, im öffentlichen Leben, die 
als positive wie negative Abhängigkeiten erlebt werden, zeigt sich im Infektionsgeschehen so banal wie brutal allein schon an der Gefahr, die von gemeinsam genutzter Luft ausgeht. Wir sind existenziell auf saubere Atemluft und damit darauf angewiesen, die Wahrscheinlichkeit einer Virusübertragung über den Luftweg zu minimieren. Niemand besitzt »Lufthoheit « - über die Atemluft sind wir alle miteinander auf einer biologischen Ebene miteinander verbunden, noch bevor wir ein Wort miteinander gesprochen haben. Atemluft ist immer soziale Atemluft. Wir erleben es als einschneidende Veränderung in allen Lebensbereichen, wenn wir den gewohnten Anteil gemeinsamer Atemluft reduzieren müssen. Größere physische Abstände führen zu größeren sozialen Abständen, die teilweise enorme Anpassungsleistungen erfordern und unser gesellschaftliches Zusammenleben stark beeinflussen.

Wie man die veränderten Distanzverhältnisse auch bewertet, keiner kann sich der durch die neuen Luftverhältnisse wieder in den Vordergrund gerückten Einsicht verwehren, wie voraussetzungsreich unsere moderne Lebensweise ist und wie stark wir auf andere angewiesen sind. Ohne verlässliche Rahmenbedingungen, zu denen wir durch unser eigenes Handeln selbst beitragen (und sei es »nur « das wohlverstandene Eigeninteresse der räumlichen Distanzierung), ist ein Ausüben individueller Freiheitsansprüche gar nicht denkbar.

Das Bewusstwerden der eigenen Verletzbarkeit ist ein wesentliches Moment für die jeweils ganz subjektive Erfahrung, warum das eigene Wohl vom Wohl der anderen abhängt und dieses wiederum beeinflusst. Keiner von uns kann im wörtlichen wie übertragenen 
Sinne im luftleeren Raum überleben und jeder füllt das »Vakuum« durch seinen Beitrag mit. Dieses außerhalb unseres persönlichen Wollens liegende systemische Aufeinander-Angewiesensein übersteigt oft unser Wahrnehmungsvermögen. Deshalb tun sich Viele schwer, die funktionale Wichtigkeit sozialer Verbundenheiten (einschließlich ihrer biologischen Vorbedingungen) als Voraussetzung für individuelle Freiheiten zu erkennen. Für so manchen wird erst im Krisenfall offenbar, wie jede Form von Selbstverwirklichung durch erlebte Autonomie, Selbststeuerung und Selbstwirksamkeit sich als fragiles Ergebnis einer höchst kollektiven Leistung erweist: Ohne Gemeinwohl keine Freiheit, ohne Wir kein Ich - wir »sind « nur durch andere und mit anderen. Ich denke, diese augenöffnende Erfahrung eines Primats des Sozialen ist die Hauptaussage der Anmerkung von Boris Johnson und weniger die Frage nach der Rolle des Staates und der Verantwortung des Einzelnen.

\section{Die Renaissance des Gemeinwohldenkens}

Das Gemeinwohldenken erfährt nicht erst seit der aktuellen Pandemie einen Aufschwung. Seit der Finanzkrise 2008/9 und mit den immer deutlicher heraufziehenden Klimaszenarien hat die individualistische Denkweise bereits einen Knacks bekommen. Die Corona-Krise gibt der Gemeinwohlorientierung eine noch stärkere, vielleicht sogar jähe Wendung.

Sie könnte die aktuellen Antworten auf die Frage nach dem Gemeinwohl sogar in einem Ausmaß erschüttern, wie das Erdbeben von Lissabon im Jahre 
I755 die Philosophen aufgerüttelt hat, neu und anders über die Rolle des Menschen in seinen Verbundenheiten mit der Welt nachzudenken. Im 2 I. Jahrhundert geht es nicht mehr in erster Linie darum, wie ein gütiger Gott ein Unheil zulassen konnte (Theodizee-Problem). Die heutige Frage ist, wie die moderne, offene Gesellschaft auf Krisen reagieren sollte, die nur durch massive Einschränkungen persönlicher Freiheiten überwunden werden können. Wie ist dann das Verhältnis von Gemeinwohl und Freiheit zu denken?

Gemeinwohl ist kein Gegensatz zur Freiheit eines jeden und aller, sondern deren Voraussetzung - ganz im Sinne des Zweiten Vatikanischen Konzils. Dort wurde Gemeinwohl gefasst »als die Gesamtheit jener Bedingungen des gesellschaftlichen Lebens, die sowohl den Gruppen als auch deren einzelnen Gliedern ein volleres und leichteres Erreichen der eigenen Vollendung ermöglichen. $\ll^{2}$

$\mathrm{Zu}$ dieser Systemperspektive kommt die lebensweltliche Sicht, nach der Gemeinwohlerfahrung zu verstehen ist als

generalisierte Sozialerfahrung in Form von intuitivem Wissen über die Sozialverhältnisse [...] Gemeinwohl ist für jeden von uns wichtig, weil wir uns nicht einfach eine Welt erfinden können, sondern vieles bereits vorfinden, was für uns alle bedeutsam ist. Gemeinwohl steckt sozusagen auch in den Rahmenbedingungen, die kollektiv gesetzt werden. Es sind Ermöglichungsbedingungen in einem Beziehungsfeld, die unserer Identität und Selbsterzählung Richtung und Struktur geben. ${ }^{3}$ 
Diese im Grunde urliberale Denktradition hatte es allerdings nicht leicht in den letzten Dekaden und die Gemeinwohlskepsis hatte Konjunktur. Klug geworden aus der Erfahrung des Dritten Reiches, wurde oft das Kind mit dem Bade ausgeschüttet und die ursprünglich wohlbedachte Zurückhaltung ungeniert mit Autoritätsverweisen (vor allem mit Carl Schmitts Diktum: »Wer bonum commune sagt, will betrügen « $)^{4}$ aufgeladen. Zusammen mit der fortschreitenden funktionalen Differenzierung wird der Kern des Gemeinwohldenkens immer weiter ausgehöhlt und verdampft scheinbar $\mathrm{zu}$ individuellen Lebensstilen (»Singularitäten«). ${ }^{5}$

$\mathrm{Ob}$ nun in der Privatisierungswelle von Einrichtungen der Daseinsvorsorge, in Verwaltungsreformansätzen oder in der Wirtschaftswelt, überall war man der Überzeugung, das Gemeinwohl stelle sich am ehesten ein, wenn ökonomische und insbesondere wettbewerbliche Maximen zum Tragen kommen. Wenn jeder an sich denkt, ist für alle gesorgt - dies war das Mantra in der Tradition von Adam Smith, dem schottischen Moralphilosophen, dessen Sicht ganz zu Unrecht auf die »unsichtbare Hand « der Marktkräfte verkürzt wurde. Gemeinwohlverweigerer sahen in der modernen Gesellschaft keine verbindenden systemrelevanten Qualitäten mehr und wollten gar der Wirtschaft eine Gemeinwohlverpflichtung wegphilosophieren. Dies dürfte sich als kurzsichtig erweisen.

Welche Rolle Gemeinwohlfragen in der Bevölkerung spielen, verdeutlicht regelmäßig der GemeinwohlAtlas, eine repräsentative Befragung zu Themen des Gemeinwohls. So waren 2019 in Deutschland 8I Prozent der Befragten besorgt, dass dem Gemeinwohl im Land 
nicht genügend Aufmerksamkeit geschenkt werde. ${ }^{6}$ Der Bedarf, das Verbindende in der Gesellschaft wieder stärker in den Blick zu nehmen, ist keineswegs allein eine akademische Debatte.

Jenseits differenzierungstheoretischer Akzente: Umkehrung des Ausgangspunktes

Kommt nun etwas ins Rutschen? Die Antwort muss in der Art und Weise unseres Zusammenlebens gesucht werden und führt dann schnurstracks zur Gemeinwohlfrage, die auf Erden und nicht im Himmel beantwortet werden will. Zur Disposition steht nicht weniger als die Umkehrung des Ausgangspunktes, wonach nicht die funktionale Differenzierung, sondern die sozialen Verbundenheiten die Basis für das moderne Gemeinwesen bilden. Es ist die Qualität der Verbundenheiten zwischen den Menschen, auf der jede Gesellschaftsordnung aufbaut und aus der sie ihre legitimierende Kraft bezieht. Von dort kommt auch der notwendige Rückhalt, der eine soziale Ordnung erst überlebensfähig macht. Wir sehen jetzt, wie stark zum Beispiel unser Wirtschaftssystem von diesen Verbundenheiten abhängt und ohne diese nicht funktionieren kann.

Die gegenseitige soziale Bejahung, das Primat des Sozialen, baut selbst auf psychologischen und, wie wir nun sehen, auch biologischen Verbundenheiten auf, die uns in einem viel stärkeren Ausmaß voneinander abhängig machen, als uns lieb sein kann. Das Soziale bildet den Rahmen, in dem sich Individualitätsformen erst entwickeln können. 
Man darf daher vermuten, dass durch COVID- I 9 beschleunigt die hohe Zeit der Differenzierungstheorien $\mathrm{zu}$ einem Ende kommt und Integrationstheorien wieder Konjunktur haben. Gesellschaften sind demnach weniger anhand der Differenzierungen, Singularitäten oder Eigenlogiken zu beschreiben, sondern vielmehr und stärker anhand der sie prägenden Verbundenheiten. Was lange nicht ins Bewusstsein gehoben werden musste, wird nun deutlich: Differenzierungen muss man sich leisten können. Wo diese zu groß werden und das Verbindende unterminieren, nimmt das Gemeinwohl Schaden.

Mit der Umkehrung des Ausgangspunktes kommt ein weiterer Gedanke ins Spiel: Entdifferenzierung bedeutet Rückführung auf allgemeinere Fragen der Verbundenheit und damit Komplexitätsreduktion. Dieses Wechselspiel von Differenzierung und Entdifferenzierung benötigt gemäß der Entwicklungspsychologie nach Jean Piaget ein dynamisches Gleichgewicht der Einordnung von neuen Erfahrungen in bestehende Weltbilder (Assimilation) und Veränderung dieses Weltbildes selbst durch neue Erfahrungen (Akkommodation). 7 Die »Zumutungen « der Perspektivenvielfalt in der Moderne ${ }^{8}$ erzwingen geradezu neue Formen der akkommodierenden Komplexitätsverarbeitung und Vereinfachung. In der entstehenden neuen Qualität werden bisherige Differenzierungen auf einem neuen Niveau integriert. Was vorher schwierig und kompliziert aussah, wird plötzlich in einem neuen Licht verständlich und offenbart eine innere Ordnung, zum Beispiel ein Muster, eine Regel oder ein System. Dieser durch Jean Piaget entwicklungspsychologisch beschriebene Basismecha- 
nismus ist notwendig, um die Fülle unterschiedlicher Perspektiven in einen kohärenten Zusammenhang zu bringen, ohne den absichtsvolles Handeln nicht möglich ist. Entdifferenzierungen bieten die Chance, neue Wege einzuschlagen, wo sich vor lauter Paradoxien und Dilemmata kein Ausweg mehr aufzutun scheint.

Die zumutungsreichen Differenzierungstendenzen überfordern ansonsten schnell den menschlichen Verstand, mit der Perspektivenvielfalt umzugehen, und erweisen sich als verwirrend. Für das Gemeinwohldenken noch bedeutsamer ist aber das mit dem Differenzierungsdiskurs verknüpfte Verschleiern von fundamentalen gegenseitigen Abhängigkeiten, wie sie uns durch die Pandemie wieder bewusst werden. Damit ist nicht ein Plädoyer für einen Abbruch der Differenzierungsdebatte aufgrund einer existenziellen Gefahr gemeint, die uns eine Besinnung auf »das Wesentliche« lehrt. Vielmehr geht es darum, eine Akkommodation voranzutreiben, sodass alle Akteure gleichberechtigt am Gemeinwohl partizipieren (können).

Man könnte auch sagen: Es geht um einen neuen Blick auf das, was Friedrich Hölderlin den einzigen »Streit in der Welt « nannte, »was nämlich mehr sei, das Ganze oder das Einzelne«. Wie sich Gemeinwohldenken tatsächlich umorientieren wird, bleibt abzuwarten. Die Krise für die Durchsetzung eigener Überzeugungen (z.B. zum Klimawandel oder zur Globalisierung) nutzen zu wollen, ist sicher legitim. Dies geht im individuellen Lebensraum leichter als in einer komplexen Gesellschaft. Ein Beispiel: Auf eine Kreuzfahrt zu verzichten ist einfacher, als neue Schiffsantriebe für die gesamte Branche zu erwirken. 
Eine Lehre lässt sich aber schon jetzt ableiten: Eine Antwort, warum das Gemeinwohldenken wieder an Bedeutung gewonnen hat, liegt in der neuen Sensibilität für Verletzbarkeit im Allgemeinen. Gemeint ist nicht Hypersensibilität für Identitätsangriffe aufgrund von Anderssein oder Fremdheitserfahrungen, sondern eine neue Aufmerksamkeit für die prinzipielle Gefährdetheit des Lebens. ${ }^{9}$ Indem der Einzelne sich seiner eigenen Verletzbarkeit bewusst wird, besteht die Chance, die fundamentale Abhängigkeit von anderen zu erkennen. Diese Ethik der Verletzbarkeit ist zugleich eine Ethik des Gemeinwohls, wie sie sich widerspiegelt in der Debatte um »vulnerable Gruppen« in der Gesellschaft, die es besonders vor dem Virus zu schützen gilt.

In der Ausrichtung auf die universelle Lebensbedingung der Verletzbarkeit und damit ein Gemeinwohlprinzip liegt eine große Chance jenseits von Identitätspolitik - für eine neue Solidarität, für eine moderne Form der Barmherzigkeit und damit für eine neue Liberalität, bei der zuerst der Mensch unabhängig von Herkunft und ethnischer Zuschreibung in seinen Entfaltungsmöglichkeiten gesehen wird. Diese Rückführung auf das Menschsein an sich wäre dann ein unvermuteter Gemeinwohlfortschritt, der seinerseits wieder neue Differenzierungen und Perspektiven erlaubt. Die moderne Gesellschaft mit ihrer hochgradigen funktionalen Differenzierung wird sich so wieder ihrer Gemeinwohlbasis bewusst, die sie aus den Augen zu verlieren schien. 


\section{Aggregatzustände des Gemeinwohls}

Während in der Corona-Pandemie die Luftqualität auf neue Weise und buchstäblich existenziell bedeutsam wurde, erweist sich die Redeweise vom Gemeinwohl als geteilter Luft, die niemandem allein gehört, zu der jeder beiträgt, die jeder verschmutzen oder sauber halten kann usw., auch in allgemeiner Weise als nützlich, den sozialen und dynamischen Charakter des Gemeinwohls $\mathrm{zu}$ beschreiben. Nicht umsonst halten sich in der Alltagssprache metaphorische Formulierungen, wie etwa »sich Luft machen«, »dicke Luft« oder »dünne Luft«.

Zur Unterscheidung, was in den kommenden Debatten nur »heiße Luft « ist und wo wirklich etwas in Bewegung kommt, lohnt es sich, auf die Idee der Aggregatzustände der öffentlichen Meinung von Ferdinand Tönnies, einem der Gründerväter der Soziologie, zurückzublicken. ${ }^{\circ}$ Damit ist der griffige Gedanke verbunden, das Aufkommen und Vergehen von Gemeinwohlüberzeugungen prozesshaft zu fassen.

Auf das Gemeinwohl angewendet, lassen sich drei in ihrer Verbindlichkeit andersartige Formen unterscheiden: Festes, solides Gemeinwohl (z.B. Demokratieprinzip), flüssiges Gemeinwohl (z.B. Diskussion um neue Formen der Bürgerbeteiligung) und gasförmiges Gemeinwohl (z.B. Einfluss sozialer Medien auf die kollektive Willensbildung). Je nach Zustandsform geht es um mehr oder weniger verbindliche Werte, Regeln und Normen. In einer liberalen, offenen Gesellschaft muss das Gemeinwohl in Bewegung bleiben und Phasenübergänge in alle Richtungen zulassen, solange diese innerhalb der verfassungsmäßigen Ordnung begründet 
werden können oder zu einer Anpassung derselben führen.

Während wir in normalen Zeiten uns eher mit luftartigen und flüssigen Formen des Gemeinwohls beschäftigen und (feine) Unterschiede kultivieren können, kommen in Krisensituationen dessen festere Bestandteile umso deutlicher zum Vorschein. Tragen diese nachhaltig oder sind sie auf Sand gebaut? Zerstört das auch nur temporäre Außerkraftsetzen von verrechtlichten Gemeinwohlüberzeugungen deren innere Bejahung? Was passiert, wenn sich bisherige Selbstverständlichkeiten individueller Freiheit als kontraproduktiv für das kollektive Überleben erweisen?

Es sind die breit akzeptierten und stabilen Gemeinwohlgrundlagen, ohne die wir uns die Oberflächendifferenzierungen (Singularitäten, Filterblasen, Echokammern) gar nicht leisten können, wenn das Überleben auf dem Spiel steht. Umgekehrt birgt die Überhöhung einzelner scheinbar in Stein gemeißelter Werte genau die Gefahr ihrer Zerstörung. Der Zweck hat noch nie die Mittel geheiligt. Jetzt wird sichtbar, wie es um das Gemeinwohl wirklich steht, ob aufgebrochene Routinen des Alltagslebens in Partnerschaften, in Familien, in den Unternehmen und Behörden, im Grunde in allen Bereichen des Zusammenlebens wieder greifen oder irreversible Veränderungen eintreten und völlig andere Verbundenheiten und damit Gemeinwohlkonstellationen entstehen.

So ist offen, ob die aus der Not vorangetriebenen technologieunterstützten Interaktionsformen sich verfestigen oder lediglich eine temporäre Verflüssigung von vorher lockeren Verbindlichkeiten sind. Der weit- 
aus größte Härtetest für das Gemeinwohl dürfte aber die Frage sein, ob die durch die Krise sich möglicherweise verschärfenden Diskrepanzen zwischen sozial, kulturell und ökonomisch Privilegierten und allen anderen zu Unzufriedenheit, sozialen Konflikten und Entfremdung führt, die dann auch Steine ins Rollen und die Verhältnisse zum Tanzen bringen kann.

Das genannte Beispiel der Frage nach Veränderungen in der kollektiven Willensbildung (Demokratieprinzip, neue Formen der Bürgerbeteiligung, Einfluss sozialer Medien auf die kollektive Willensbildung) ist eine nach veränderten Gemeinwohlkonstellationen, d.h. dem Potenzial zur Vermittlung individueller Bedürfnisbefriedigung und funktionierendem Gemeinwesen im Bereich von Teilhabe und Teilnahme an gesellschaftlichen Prozessen.

Gemeinwohlkonstellationen auf ihren Aggregatzustand $\mathrm{zu}$ befragen, hat einen ganz praktischen Wert: Der Leser dieses Beitrages nehme in einer Tageszeitung einen Kommentar zu politischen, wirtschaftlichen, kulturellen oder sozialen Fragen zur Hand und suche nach festen, flüssigen und gasförmigen Bestandteilen des Gemeinwohls. In vielen Fällen wird sich dies als erhellend erweisen, um mögliche Gemeinwohlentwicklungen zu erkennen.

Die Herausforderung besteht darin, die Veränderungen der Aggregatzustände des Gemeinwohls auf ihren Beitrag zum funktionierenden, lebendigen Gemeinwesen zu überprüfen. Entscheidungsträger stehen in Verantwortung, bei der Gemeinwohlfrage gut abzuwägen, wo sie gerade den Meißel anlegen bzw. etwas verfestigen möchten. Die große Deutungs- und in der 
Konsequenz Führungsleistung besteht in der Fähigkeit, das Gemeinwohl im Konkreten zu suchen, aber nicht damit zu verwechseln. Als »notwendige Fiktion« weist die Gemeinwohlerfahrung zwangsläufig über die unmittelbaren Tatsachen hinaus bzw. kann aus ihnen nicht direkt abgeleitet werden. ${ }^{\text {II }}$ Die $\gg$ Fakten « müssen erst in der gedanklichen Vorstellung auf ihre mögliche Gemeinwohlerfahrung hinterfragt werden. Diese besteht in einer inneren Bejahung oder Ablehnung von sozialen Tatsachen. Die Zuschreibung eines Gemeinwohlwertes ist keineswegs beliebig oder gar willkürlich, wenn man den Gedanken vom Primat des Sozialen ernst nimmt und subjektive Bewertungen selbst als Ausdruck von sozialen Prozessen sieht, durch die der Einzelne gesellschaftliche Werte und Normen verinnerlicht hat.

Diese Paradoxie der sogenannten immanenten Transzendenz des Gemeinwohls lässt sich auf den Punkt bringen: »Gemeinwohl als Erfahrungskategorie des Sozialen zu erfassen bedeutet zwangsläufig, auf die Wahrnehmungsfähigkeiten beim Individuum zu setzen, ja letztlich darauf setzen $\mathrm{zu}$ müssen. Damit sind nicht einfach sinnliche Eindrücke gemeint, sondern die Fähigkeit, eigene Erfahrungen mit der Sozialwelt in einen subjektiven Bedürfnishintergrund $\mathrm{zu}$ übersetzen und emotional einzuordnen. $\ll^{12}$

In der akuten Corona-Krise ist - wie bereits erwähnt - die eigene Verletzbarkeit und der Zusammenhang zwischen individuellem und kollektivem Überleben der Schlüssel für die Gemeinwohlrelevanz. Nach der Krise werden wieder andere Relevanzgesichtspunkte nach vorn rücken, wie die historische Analyse vergangener Pandemien nahelegt. ${ }^{13}$ 


\section{Postpandemisches Gemeinwohl}

Im Sommer 2020 - im ersten Jahr der Pandemie - erhobene Daten zeigen, dass sich mit der Corona-Krise das Gemeinwohlbewusstsein in der Bevölkerung verändert. So geben 60 Prozent der Befragten an, dass ihnen das Gemeinwohl wichtiger geworden sei. ${ }^{14} \mathrm{Ob}$ dieser Effekt von Dauer ist, bleibt abzuwarten.

An vielen Stellen sehen Kommentatoren bereits jetzt katalytische Wirkungen der Pandemie am Werk, die neue Entwicklungen ermöglichen oder sich bereits abzeichnende beschleunigen bzw. in eine bestimmte Richtung lenken. Steht sogar wieder einmal ein grundlegender Wandel, eine Metanoia, der Menschen zum guten Leben in Gemeinschaft und Gesellschaft an?

Für Entscheidungsträger in Wirtschaft, Politik und Gesellschaft ist die Dynamisierung des Gemeinwohls Chance und Risiko zugleich. Argumente zur Gemeinverträglichkeit, Gemeinförderlichkeit oder Gemeinwohlinnovation, aber auch Gemeinwohlverletzung und Gemeinwohlschädigung sind gut geeignet, komplexe Entscheidungen $\mathrm{zu}$ legitimieren oder $\mathrm{zu}$ delegitimieren. Ohne Zweifel wird diese Art der Begründungserleichterung oder auch Begründungsbeschwerung in den nächsten Jahren zentrale Diskurse in der Öffentlichkeit bestimmen. Der tiefere Grund dafür liegt in der Integrationskraft des Gemeinwohldenkens, um entstehende Widersprüche und Paradoxien durch Verlagerung auf eine andere Bezugsebene zu überwinden. Nicht ein Noch-Mehr an Reflexion, sondern eine attraktive Kombination von gedanklicher Reflexion und emotionaler Ansprache ist dann das Erfolgsrezept. Gemeinwohl 
kann nie wahr oder falsch sein - es geht immer um die Durchsetzung bestimmter Werthaltungen, die sich subjektiv bewähren und kollektive Bejahung erfahren.

In Krisensituationen entstehen immer wieder Scheidewege, an denen es im aufgeklärten Europa bei aller Bewegungsfreiheit des Gemeinwohls nur einen Kompass geben kann: die Unantastbarkeit der Würde des Einzelnen. Insofern sollte jetzt die Zeit der liberalen Gemeinwohldenker anbrechen, die sich die Voraussetzungen für die freie Entfaltung eines jeden und damit aller zur Denkmaxime machen. Die Gemeinwohlfrage wird zur liberalen Frage schlechthin.

\section{Anmerkungen}

I Boris Johnson, Videobotschaft, https://t.co/kxdqItMYSE, Twitter, 29. März 2020, abgerufen am 30. I. 202 I.

2 Zweites Vatikanisches Konzil, Pastorale Konstitution, Gaudium et spes. Über die Kirche in der Welt von heute, Rom: Acta Apostolicae Sedis 58, I966.

3 Timo Meynhardt, Ohne Gemeinwohl keine Freiheit: Zur Psychologie des Gemeinwohls, in: Freiheit und Gemeinwohl: Ewige Gegensätze oder zwei Seiten einer Medaille?, hg. von Hans-Jürgen Papier und Timo Meynhardt, Hamburg 20I6, S. I 88 .

4 Zitiert nach: Josef Isensee, Gemeinwohl und öffentliches Amt. Vordemokratische Fundamente des Verfassungsstaates, Wiesbaden 2013 , S. 39.

5 Andreas Reckwitz, Die Gesellschaft der Singularitäten: Zum Strukturwandel der Moderne, Berlin 2018.

6 www.gemeinwohlatlas.de, abgerufen am 30. I. 202 I.

7 Jean Piaget, Das Erwachen der Intelligenz beim Kinde. Stuttgart 2003 .

8 Peter Strohschneider, Zumutungen: Wissenschaft in Zeiten von Populismus, Moralisierung und Szientokratie, Hamburg 2020.

I 76 
9 Judith Butler, Gefährdetes Leben. Politische Essays, Frankfurt a.M. 2005 .

I० Ferdinand Tönnies, Kritik der öffentlichen Meinung, Berlin/ Heidelberg I $922 / 2013$

I I Timo Meynhardt, Public value inside: What is public value creation?, in: International Journal of Public Administration, 32. Jg., Heft 3-4, 2009, S. I92-2 I9.

I 2 Timo Meynhardt (Anm. 3), S. I 80.

I 3 Laura Spinney, I91 8 - Die Welt im Fieber: Wie die Spanische Grippe die Gesellschaft veränderte, München 2018.

I4 www.gemeinwohlatlas.de, abgerufen am 30. I. 202 I. 